\title{
Theranostic imaging of liver cancer using targeted optical/MRI dual-modal probes
}

\author{
Qingshan Chen ${ }^{1,2, *}$, Wenting Shang ${ }^{2,3, *}$, Chaoting Zeng ${ }^{1,2}$, Kun Wang ${ }^{2,3}$, Xiaoyuan \\ Liang ${ }^{2,3}$, Chongwei Chi ${ }^{2,3}$, Xiao Liang ${ }^{2,3}$, Jian Yang ${ }^{1,2}$, Chihua Fang ${ }^{1}$ and Jie Tian $^{2,3}$ \\ ${ }^{1}$ Department of Hepatobiliary Surgery, Zhujiang Hospital, Southern Medical University, Guangzhou 510280, China \\ ${ }^{2}$ Key Laboratory of Molecular Imaging, Institute of Automation, Chinese Academy of Sciences, Beijing 100190, China \\ ${ }^{3}$ Beijing Key Laboratory of Molecular Imaging, Beijing 100190, China \\ "These authors have contributed equally to this work \\ Correspondence to: Chihua Fang, email: fangch_dr@126.com \\ Jie Tian, email: jie.tian@ia.ac.cn \\ Keywords: liver cancer, MRI/optical, preoperative diagnosis, intraoperative navigation, dual-modality \\ Received: May 12, $2016 \quad$ Accepted: December 05, $2016 \quad$ Published: February 23, 2017 \\ Copyright: Chen et al. This is an open-access article distributed under the terms of the Creative Commons Attribution License (CC-BY), \\ which permits unrestricted use, distribution, and reproduction in any medium, provided the original author and source are credited.
}

\section{ABSTRACT}

The accurate preoperative detection and intraoperative navigation afforded by imaging techniques have had significant impact on the success of liver cancer surgeries. However, it is difficult to achieve satisfactory performance in both diagnosis and surgical treatment processes using any single modality imaging method. Here, we report the synthesis and characteristics of a novel dual-modality magnetic resonance imaging (MRI) and near-infrared fluorescence (NIRF) probe and verify its feasibility in nude mouse models with liver cancer. The probes are comprised of superparamagnetic iron oxide (SPIO) nanoparticles coated with liposomes to which a tumor-targeted agent, Arg-Gly-Asp peptides (RGD), and a NIRF dye (indocyanine green, ICG) have been conjugated. Specific targeting, biodistribution, and the imaging ability of the probes for MRI-NIRF were examined. Furthermore, we applied the dual-modality methodology toward the preoperative diagnosis and intraoperative guidance of radical resection in mouse models with both orthotopic liver tumors and intrahepatic tumor metastasis. The study demonstrated that both MRI and fluorescent images showed clear tumor delineation after probe injection (SPIO@Liposome-ICG-RGD). The contrast-to-noise ratio obtained from MRI was $31.9 \pm 25.4$ at post-injection for the preoperative diagnosis, which is helpful for detecting small tumors $(0.9 \pm 0.5$ $\mathrm{mm}$ ). The maximum tumor to background ratio of NIRF imaging was $2.5 \pm 0.3$ at $72 \mathrm{~h}$ post-injection for effectively capturing miniscule tumor lesions $(0.6 \pm 0.3 \mathrm{~mm})$ intraoperatively. The novel MRI-NIRF dual modality probes are promising for the achievement of more accurate liver tumor detection and resection.

\section{INTRODUCTION}

Liver cancer is one of the most common malignant cancers worldwide with an estimated incidence of 782,500 cases in 2012 , of which roughly half arose in China [1]. Approximately $40 \%$ of patients with liver cancer are diagnosed in the early stage and have a 5-year survival rate of about $28 \%$. Survival decreases to $10 \%$ and $3 \%$ for patients who are in the regional and distant stages of the disease, respectively [2]. Therefore, there is an urgent need for accurate preoperative diagnosis and assessment, along with optimization of the treatment scheme.

Surgical resection is one of the major therapeutic methods for liver cancer; however, its 5-year risk of recurrence exceeds $70 \%$ [3], with micro-vascular invasion and micro-residual lesions representing the main risk factors for postoperative recurrence [4]. Therefore, it is vital for surgeons to realize radical resection to facilitate patient recovery. 
Diverse imaging modalities for liver cancer currently exist, such as positron emission tomography-computed tomography (PET-CT), magnetic resonance imaging (MRI), and fluorescence molecular imaging (FMI), each of which carries specific advantages and disadvantages. However, no single imaging modality has to date been able to simultaneously provide the entirety of preoperative structural information and intraoperative local functional information for liver cancer required in clinical practice.

MRI is commonly applied in clinical liver cancer diagnosis owing to its excellent soft tissue contrast and spatial resolution [5]. Intraoperative near-infrared fluorescence (NIRF) imaging is also frequently applied for the guidance of various tumor resections because of its unique sensitivity and real-time ability [6]. Therefore, the development of an MRI-NIRF dual modality molecular imaging probe might have the potential to combine the advantages of both imaging technologies and enable better theranostic performance for patients with liver cancer.

In our research, we synthesized and tested a novel MRI-NIRF molecular imaging nanoprobe, SPIO@ Liposome-ICG-RGD. The arginine-glycine-aspartic acid (RGD) peptide can bind specifically to the integrin $\alpha v \beta 3$ receptor, which is the most important integrin for angiogenesis [7], which in turn plays a crucial role in the pathological development of many solid tumors including liver cancer [8]. The superparamagnetic iron oxide nanoparticles (SPIO NP) have the characteristics of lower toxicity, high sensitivity, and excellent biocompatibility, and were the first T2 contrast agent for MRI to be used clinically [9]. SPIO, as for the commercial product Feridex ${ }^{\circledR}$, was approved by the U.S. Food and Drug Administration (FDA) for the diagnosis of liver lesions [10] and are selectively taken up by Kupffer cells in the liver, wherein rapid dephasing of neighboring proton spins leads to a shortening of the spin-spin (T2) relaxation times. In contrast, liver tumors that lack Kupffer cells cannot take up the SPIOs effectively. Therefore, T2-weighted images of normal tissues show darkening [11]. Indocyanine green (ICG) is the only near infrared reflection (NIR) organic dye approved by the FDA for human medical imaging and diagnosis in clinical practice [12].

Here, we found that the MRI and fluorescence images showed clear tumor delineation at post-injection and $72 \mathrm{~h}$ post-injection, respectively. Therefore, the targeted dual-modality imaging probe was conducive to improving preoperative surgical planning and intraoperative fluorescence-guided surgery.

\section{RESULTS}

\section{Characterization of SPIO@Liposome-ICG-RGD}

The morphology and size of the SPIO@LiposomeICG-RGD probes were characterized using transmission electron microscopy (TEM) and dynamic light scattering, respectively. The TEM image revealed that the typical spherical morphology of the probe exhibited an approximate average diameter of $126 \pm 21 \mathrm{~nm}$ (Supplementary Figure 1). Thus, the size $(<150 \mathrm{~nm})$ of the probe is particularly favorable for tumor-targeting in vivo [13]. Spectroscopic measurement of the probe showed that the NIR signal peaked at an emission wavelength around $810 \mathrm{~nm}$. With constant SPIO $(250 \mathrm{mg} / \mathrm{mL})$, different supplied amounts of ICG $(25-150 \mu \mathrm{g} / \mathrm{mL})$ yielded a similar absorbance between $780 \mathrm{~nm}$ and $830 \mathrm{~nm}$ in the UV-vis absorption spectra, which revealed that the probe was suitable for NIRF imaging (Supplementary Figure 2). With the increase in the probe concentration, the T2-weighted MRI signal increased gradually (negative contrast effect) and the optical signal also increased (Figure 1A). Both signals types showed a linear correlation (MRI: $r 2=0.9954$; NIRF: $r 2=0.9962$ ) with the probe concentration (Figure $1 \mathrm{~B}$ and Figure 1C). This suggested that the fluorescence was protected from the bleaching of SPIO because of the interval produced by the liposome. The relaxivity of the probe was $363.4 \mathrm{mM}^{-1}$ $\mathrm{s}^{-1}$, which was approximately 3 times better than that of the FDA-approved drug Feridex ${ }^{\circledR}$ for the detection of liver lesions [14]. No significant decrease of cell viability was observed when cells were incubated with any of the three NPs at a concentration range from $0-100 \mu \mathrm{g} / \mathrm{mL}$ ( $\mathrm{Fe}_{3} \mathrm{O}_{4}$ concentration) (Supplementary Figure 3), which demonstrated their good biocompatibility. Furthermore, the optical stability assessment indicated that the probe was stable in mosue serum over $96 \mathrm{~h}$ (Supplementary Figure 4 and Supplementary Figure 5). In the integrin binding assays, the targeted probe showed a $4.1 \pm 1$.1-fold $(\mathrm{P}<$ $0.05)$ increase in the uptake of SPIO when incubated with HepG2 cells (Supplementary Figure 6A) and a $2.6 \pm 0.5$ fold increase in fluorescence intensity $(\mathrm{P}<0.05)$ compared with the non-targeted control probe (Supplementary Figure $6 \mathrm{~B})$. This double verification confirmed the specific targeting ability of the targeted probes. Together, these results indicated that the optical/MRI probe was able to function efficiently in dual modality imaging.

\section{Evaluation of MRI contrast enhancement in vivo}

The quantification of MRI contrast SI between the liver and the tumor was acquired by calculating the contrast-to-noise ratio $(\mathrm{CNR})$ as $\mathrm{CNR}=\left(\mathrm{SI}_{\text {liver }}-\mathrm{SI}_{\text {tumor }}\right)$ ) $\mathrm{SI}_{\text {noise }}$ [14]. In vivo $\mathrm{MR}$ imaging was performed prior to and after injection, respectively. For mice receiving the targeted probe, the spatial distribution of the decreased pixels was heterogeneous and occurred mainly in the periphery of the tumor (Figure 2A). For mice receiving the non-targeted probe, the pixel signal decrease was much less pronounced and scattered within the tumor rim (Figure 2B). The decreased signal in the tumors was $24.7 \pm 1.5 \%$ after the administration of the targeted probe, whereas only a $5.3 \pm 2.0 \%(\mathrm{P}<0.05)$ decrease was observed in the non-targeted group. In the orthotopic liver tumor nude mouse model $(\mathrm{n}=5)$ (Figure 3), we found marked signal reductions in the normal liver tissue 
following the injection. The maximum CNR was about $31.9 \pm 25.4$ at post-injection. To our knowledge, the CNRs were found to be only $146.6 \pm 18.9$ for Feridex ${ }^{\circledR}[10]$. These data indicated that MRI scanning should be performed after the injection of the probes for preoperative assessment.

\section{In vivo optical imaging and histology examination}

After continuous observation over $120 \mathrm{~h}$, fluorescence imaging indicated a significant accumulation of both probes in tumor lesions up to $120 \mathrm{~h}$. However, the SPIO@Liposome-ICG-RGD probe demonstrated better optical contrast than the non-targeted probe in the tumor region starting at $24 \mathrm{~h}$ post-injection (Figure 4A-4B). After the initial distribution period $(<12 \mathrm{~h})$, the majority of the optical signals were observed in the tumor and abdominal areas and gradually faded away $24 \mathrm{~h}$ post-injection. These results suggested that the probes were eliminated through the liver and spleen, which aligned with our expected 100 $150 \mathrm{~nm}$ probe size [13]. Furthermore, the quantification of radiant efficiency showed that SPIO@Liposome-ICGRGD displayed a stronger optical signal in tumor lesions at all observation points (Figure 4C), verifying its excellent in vivo targeting ability for integrin $\alpha \mathrm{v} \beta 3$-overexpressing tumors. Notably, the tumor-to-background ratio (TBR) peak of SPIO@Liposome-ICG-RGD (2.6 \pm 0.1$)$ appeared at $72 \mathrm{~h}$ post-injection and was about 2-fold higher than that of SPIO@Liposome-ICG (1.3 \pm 0.1$)$ at the corresponding time (Figure 4D). The ex vivo comparisons of the biodistribution between the targeted and untargeted probes were performed on the major organs (tumor, heart, liver, spleen, lungs, and kidneys) at $72 \mathrm{~h}$ post injection, which also demonstrated the higher tumor uptake of the SPIO@ Liposome-ICG-RGD group (Supplementary Figure 7). These data confirmed SPIO@Liposome-ICG-RGD tumor specificity and indicated that intraoperative navigation should be performed $72 \mathrm{~h}$ following probe injection.

\section{Dual-modality imaging using the SPIO@ Liposome-ICG-RGD probe detects orthotopic liver tumors}

Orthotopic liver tumor nude model mice $(n=5)$ were established by two experienced surgeons. We initially performed MRI scanning to determine the characteristics of the primary liver tumors including the location, size, and number. A marked MRI signal decrease was observed in normal liver tissue subsequent to probe delivery (Figure 5A-5B). The maximum CNRs were found to be $34.2 \pm 9.1$ after the injection of SPIO@Liposome-ICGRGD. Via intraoperative fluorescence navigation through
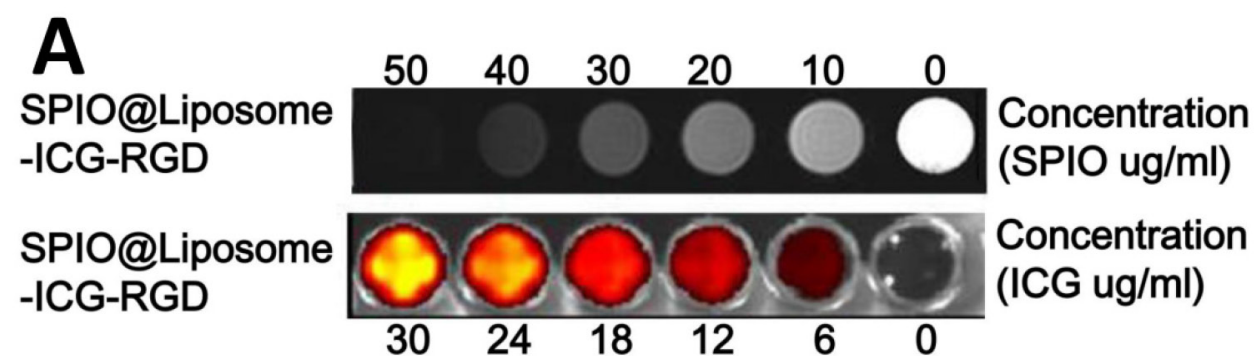

Concentration (ICG ug/ml)
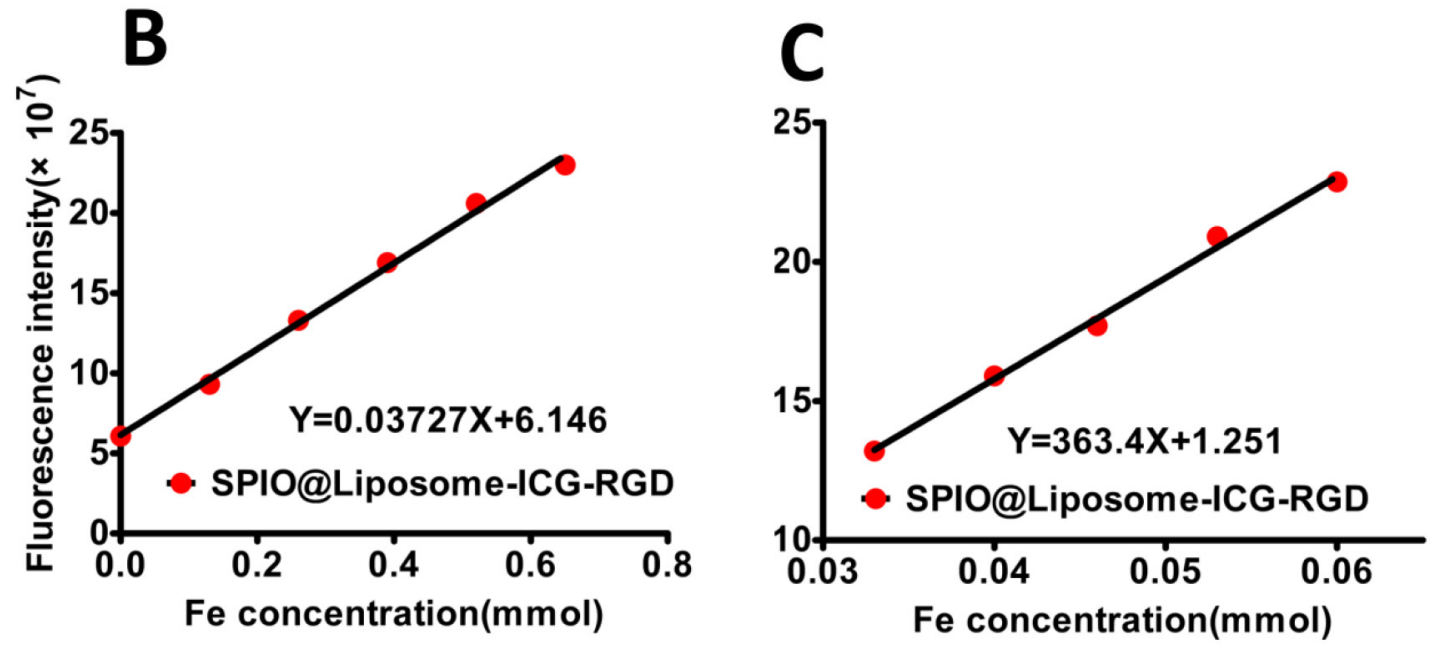

Figure 1: MRI and NIRF signal evaluation for SPIO@Liposome-ICG-RGD. A. The concentration of the probe was successively diluted from the left to right wells. For MRI, the negative contrast effect is clearly demonstrated with the decrease of SPIO concentration. However, for NIRF imaging, the optical signal decreases with diminishing ICG concentration. B. A linear correlation is observed between the fluorescence intensity and the probe concentration, whereas $\mathbf{C}$. an inverse linear correlation can be seen between the T2-weighted MRI signal and the probe concentration. The R2 of the probe is $363.4 \mathrm{mM}^{-1} \mathrm{~s}^{-1}$. 


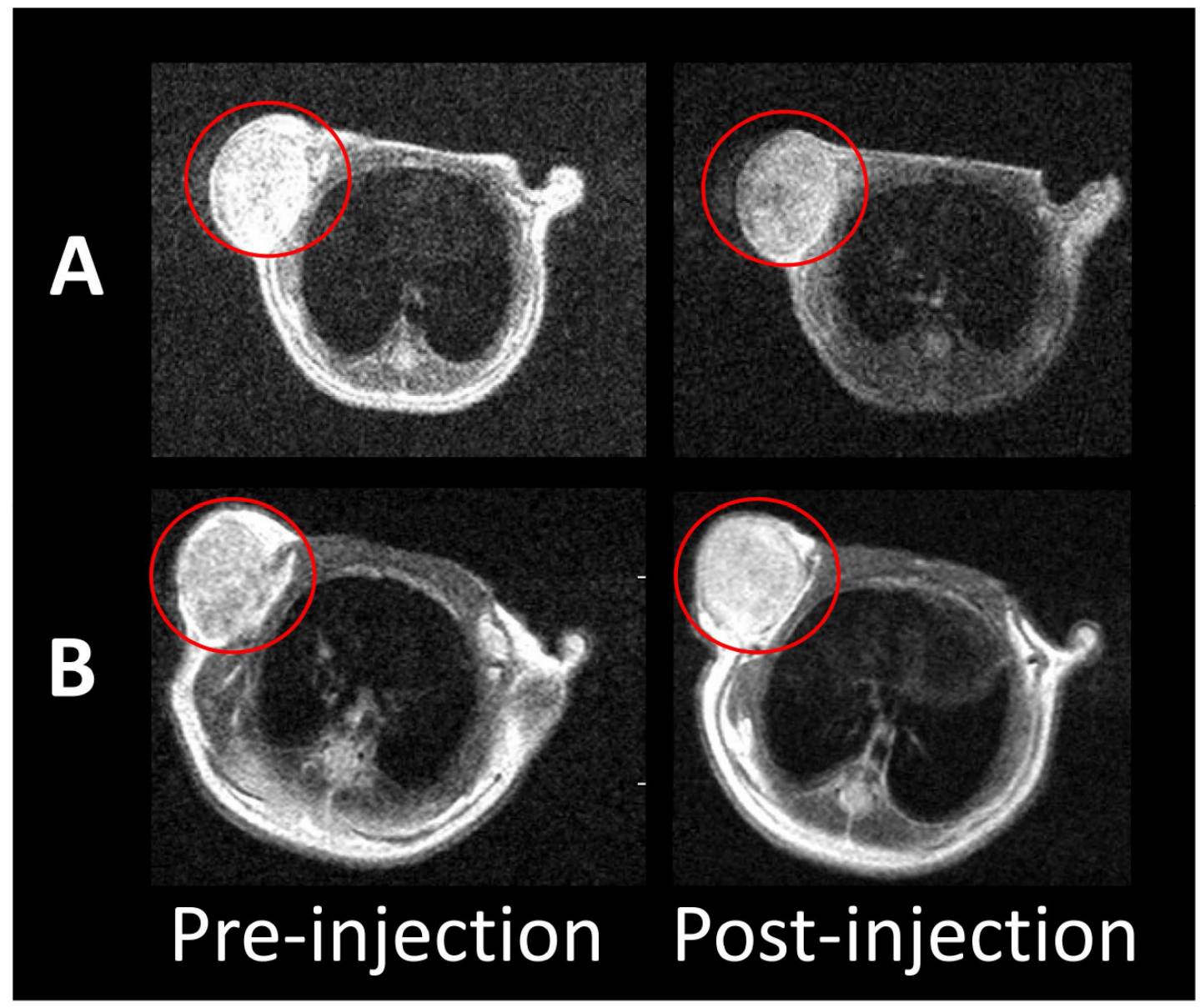

Figure 2: In vivo T2-weighted MR imaging before and after the injection of SPIO@Liposome-ICG-RGD A. or SPIO@Liposome-ICG B.

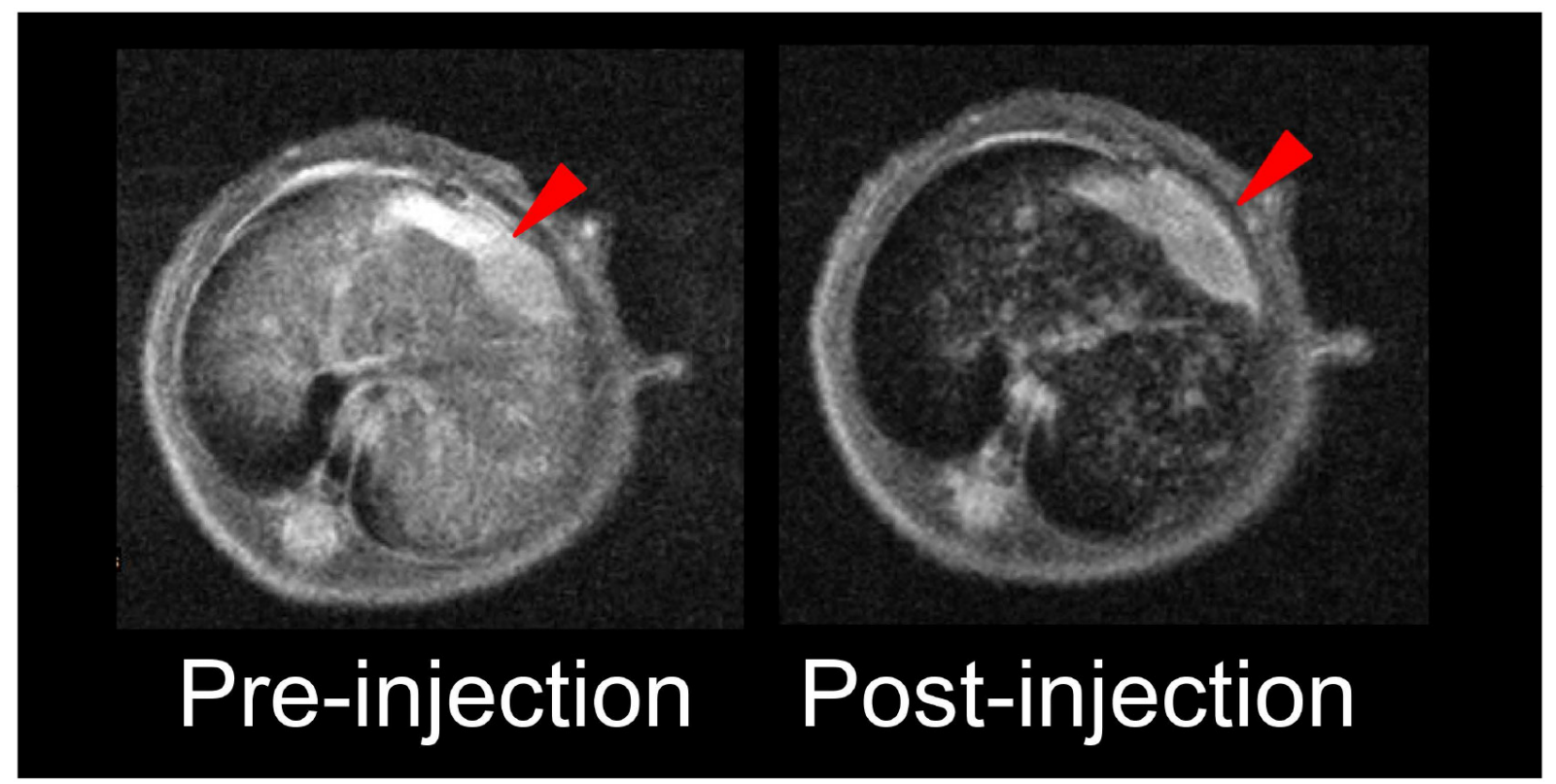

Figure 3: In vivo continuous observations of the orthotopic liver cancer model administrated with SPIO@LiposomeICG-RGD. The contrast-to-noise ratio (CNR) of SPIO@Liposome-ICG-RGD was calculated as CNR $=\left(\mathrm{SI}_{\text {liver }}-\mathrm{SI}_{\text {tumor }}\right) / \mathrm{SI}_{\text {noise }}$. 
laparotomy in another model, we observed that the fluorescent signal of the tumor was much higher than that of the normal liver tissue in the orthotopic models. Under the fluorescence surgical navigation system, we easily resected the large tumor tissue mass, which exhibited a TBR at $2.4 \pm 0.3$ fold (Figure 5C-5F). After the first operation, the miniscule residual tumor tissue $(0.6 \pm 0.3 \mathrm{~mm})$ in the surgical area was still much brighter than the normal liver tissue, which raised the concern that the operation was not sufficient to effect radical resection. We further removed the miniscule residual tumor under the optical image with the TBR at $2.5 \pm 0.3$ fold (Figure 5G-5I). Finally, the above surgical samples were confirmed by both Prussian blue staining and HE staining (Figure 5J-5K).

\section{Dual-modality imaging using the SPIO@ Liposome-ICG-RGD probe detects primary liver tumors with intrahepatic metastasis}

A total of 5 liver tumor nude mouse models with intrahepatic metastasis were established based on the orthotopic liver tumor model for extending its growing time. The preoperative MRI scanning assisted with not only detecting the large- but also the smaller tumors $(0.9 \pm 0.5 \mathrm{~mm}$ ) with a CNR of $14.6 \pm 9.9$ (Figure 6A6B). The bioluminescence imaging assisted with tumor confirmation (Figure 6C). After the preoperative assessment, we aggressively detected the tiny tumors under the fluorescence surgical navigation system. The small tumors $(0.7 \pm 0.3 \mathrm{~mm})$ were much brighter than the surrounding normal liver tissue (Figure 6D-6F) with the TBR at $2.3 \pm 0.5$. These results might have profound value in clinical practice. For example, for patients with liver cancer exhibiting similar degrees of metastasis, subsequent to MRI scanning surgeons might recommend that the patient not proceed with surgery, which might reduce their physiological and psychological stress and therefore might represent their best overall choice.

\section{DISCUSSION}

Imaging has played a vital role in the diagnosis of liver cancer. Multi-modal imaging, which can integrate the advantages of single image models, is popular with
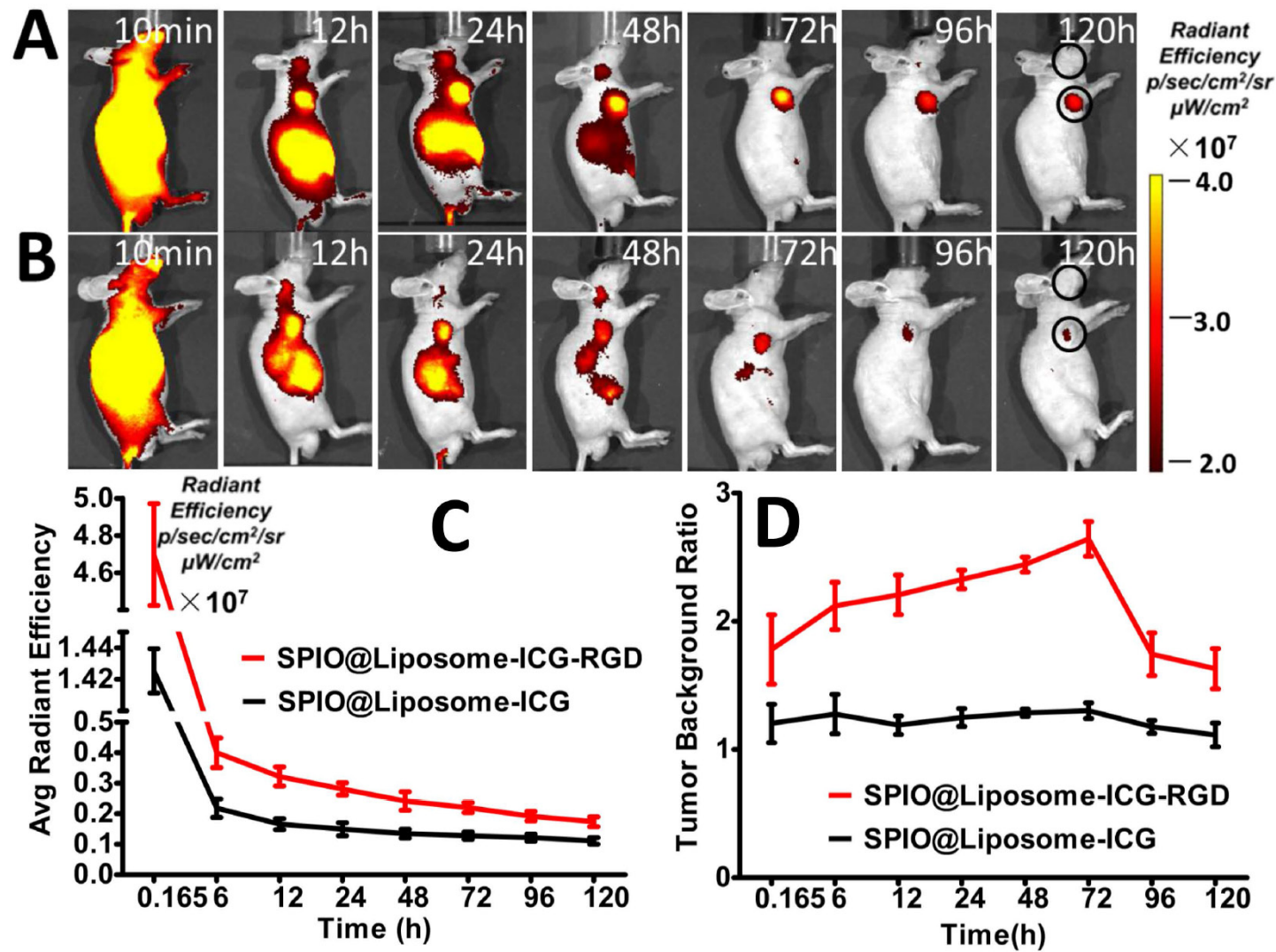

Figure 4: In vivo comparison of the biodistribution of SPIO@Liposome-ICG-RGD and SPIO@Liposome-ICG. A, B. In vivo continuous observations (120 h) of liver cancer xenografts administrated with SPIO@Liposome-ICG-RGD (A) or SPIO@, Liposome-ICG (B) using FMI. Black circles indicate the regions of interest for calculating the tumor to background ratio (TBR) in each time point. The quantification of fluorescence intensity at the tumor sites reveals a higher accumulation of SPIO@Liposome-ICG-RGD at all observation points $\mathbf{C}$. Comparison of TBR profiles of the two probes. The peak and maximum difference both occurred at $72 \mathrm{~h}$ postinjection, suggesting the optimal surgical window time D. Experiments were run in triplicate. 
researchers [15]. However, there are few successful reports on simultaneously achieving "assessment preoperatively and guided intraoperatively" for liver cancer in clinical management [16]. This strategy would allow the surgeons to understand the number, location, and size of the liver tumors preoperatively and to precisely remove them using imaging-guidance intraoperatively, which would likely greatly improve the accuracy of resection, avoid futile surgical procedures, and promote the enhanced recovery of patients with liver cancer.

For example, a CT/fluorescence probe for studying atherosclerotic plaques was reported by Ding et al. [17], and Sampath et al. synthesized a PET/near-infrared agent for staging breast cancer and guiding the subsequent intraoperative resection [18]. However, compared to the ionization radiation of CT and the high costs of PET-CT, MRI is an ideal model for acquiring overall information and optical imaging is a perfect complement for MRI to obtain real-time intraoperative data owing to its excellent sensitivity [19].

There are, however, few probes available for liver cancer. It is known that the liver is the main organ for probe elimination, which induces a nonspecific background signal of many agents and hinders the development of a probe for liver lesions. Jin et al. reported a bifunctional probe for targeting and imaging liver cancer,

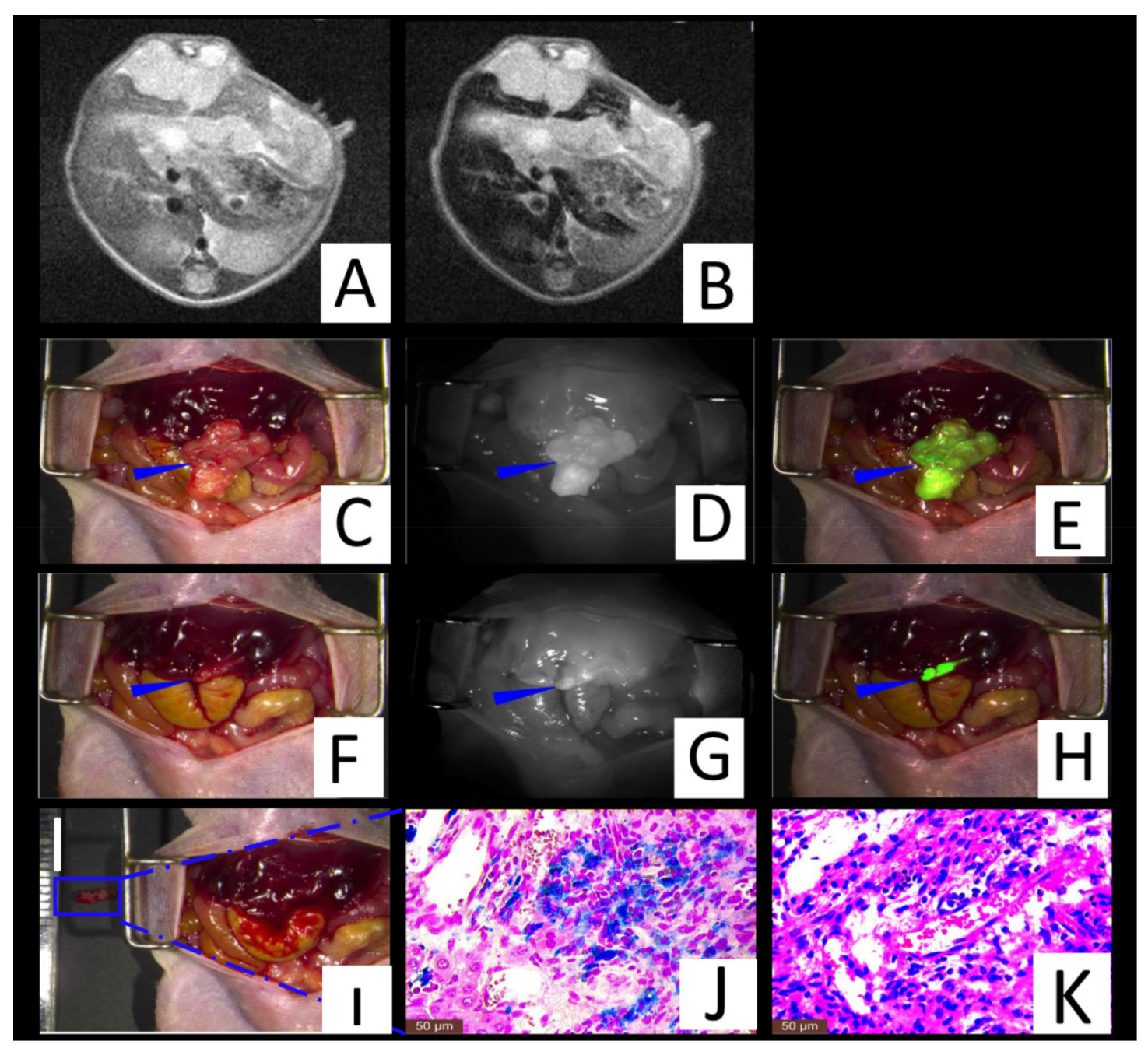

Figure 5: Theranostic imaging in the orthotopic liver tumors models. The MRI image before SPIO@Liposome-ICG-RGDs injection A. The MRI signal is obviously decreased in normal liver tissue (CNR: $34.2 \pm 9.1$ ) after targeted probe injection B. Surgical guidance by intraoperative FMI-NIR (fluorescence molecular imaging system) C. The implanted liver tumor tissue (blue arrow) exhibits obvious contrast (TBR: $2.4 \pm 0.3$ ) in color and texture with normal liver tissues $\mathbf{D}$. The merge image of color and fluorescence demonstrates the excellent contrast $\mathbf{E}$. The residual tumor node (blue arrow) after the first operation $\mathbf{F}$. The residual tumor node exhibits obvious contrast (TBR: $2.5 \pm 0.3$ ) in color and texture with normal liver tissues G. The merged color and fluorescence image demonstrates the excellent contrast in the residual tumor node $\mathbf{H}$. Identification of the residual tumor $(0.6 \pm 0.3 \mathrm{~mm})$ after the initial resection $\mathbf{I}$. Prussian blue staining confirmation of the targeting ability of SPIO@Liposome-ICG-RGDs J. HE staining confirmation of the liver tumor tissue K. Experiments were run in triplicate. 
but this was only achieved in vitro [20]. In addition, Hui et al. only achieved dual-modality imaging in heterotopic liver tumors [21].

SPIO can be absorbed to a large degree by Kupffer cells in normal liver tissues; however, liver tumors lacking these cells contain few probes despite attaining the targeting effect after injection. These probes at tumor sites were unable to provide adequate MRI signals; however, along with the metabolism of the probes by the liver; the provided signal was sufficient for fluorescence detection owing to the extreme sensitivity of optical imaging. Thus, in our research, we combined the spatial resolution of MRI and the excellent sensitivity of optical imaging, utilized the advantages of the characteristics of liver metabolism, and undertook MRI imaging and optical imaging at different time points. To our knowledge, this is the first study to successfully describe the advantages of the characteristics of probe metabolism by the liver and achieve the "assessment preoperatively and guided intraoperatively" in liver cancer models.

In this study, we conducted MRI and fluorescence experiments both in vitro and in vivo. For the specific targeting test, we verified a marked increase in the cellular uptake of ICG as measured by the fluorescence intensity in the targeted probe group, as well as a marked increase of SPIO as determined ICP-MS. These experiments verified the efficacy of our probes in specially targeting the $\alpha v \beta 3$ receptor of HepG2 cells. These observations can be explained as follows: upon specific binding of the RGD-peptide to integrin $\alpha v \beta 3$, the RGD-containing nanomaterials would be internalized into the cells by receptor-mediated endocytosis. The RGD peptide then would combine with the peptide ligand for neuropilin-1, giving rise to selective tumor vascular targeting and facilitating the penetration of imaging agents into the tumor $[22,23]$. The linear relationship between the probe concentration and the optical intensity indicated that ICG was successfully loaded into the lipid layer of the SPIO@ Liposome. The R2 relaxivity for the SPIO@LiposomeICG-RGD was $363.3 \mathrm{mM}^{-1} \mathrm{~s}^{-1}$, which was superior to the $243.3 \mathrm{mM}^{-1} \mathrm{~s}^{-1}$ of the commercial MRI agent Feridex ${ }^{\circledR}[10]$. We propose that the T2 relaxivity of the PEGylated SPIO@Liposome nanoparticles was amplified by the contribution of water exchange on the transverse relaxation time owing to the effect of PEG attached to the bilayer and in the SPIO coating [24]. For the in vivo MRI experiment, the maximum CNR was $31.9 \pm 25$ at post-injection. The occurrence of the maximum CNR at this time point was mainly the consequence of the sensitivity of MRI scanning and the tendency of our probe

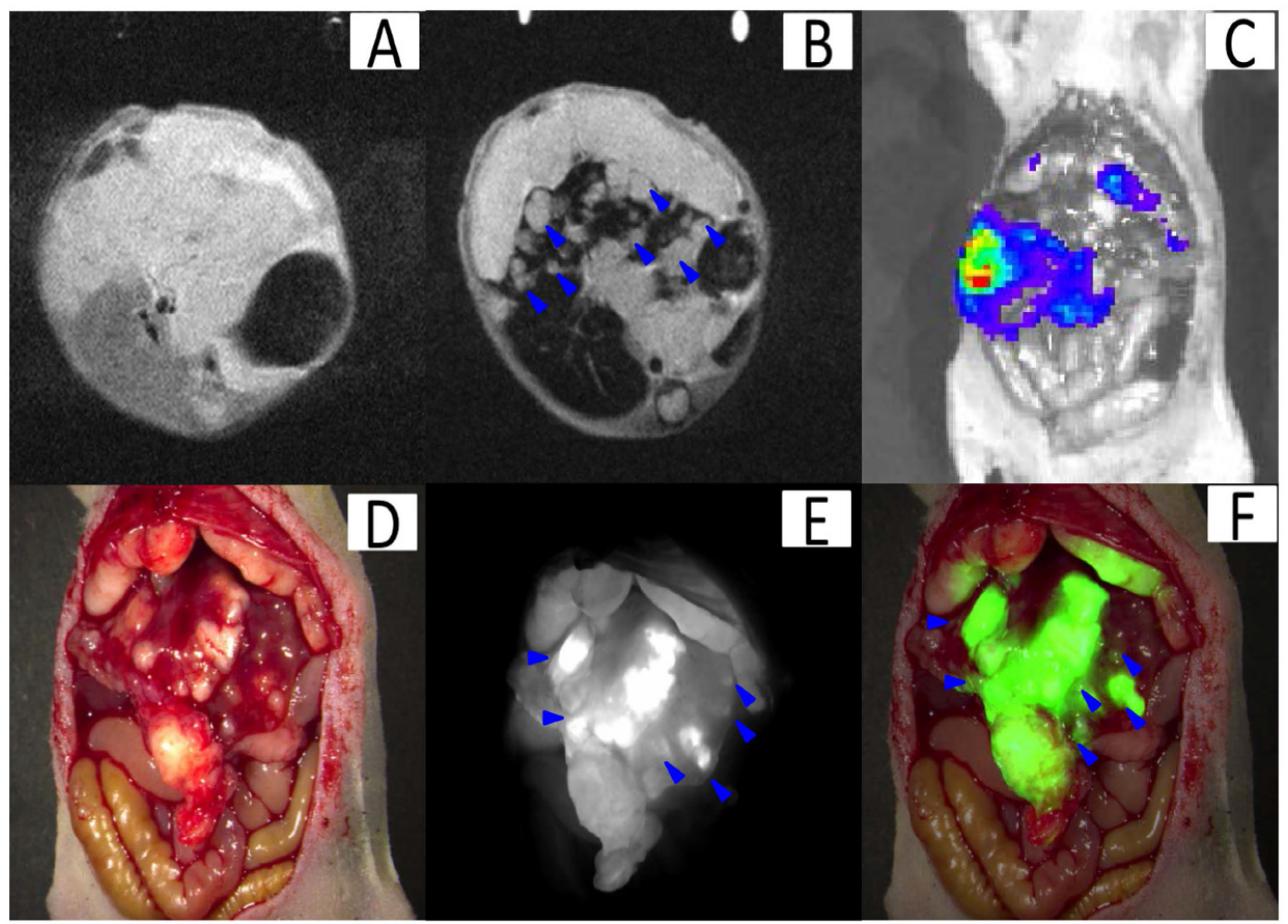

Figure 6: Theranostic imaging in the orthotopic liver tumors with intrahepatic metastasis. The MRI image before SPIO@ Liposome-ICG-RGDs injection A. The MRI signal is obviously decreased in normal liver tissue (CNR: 14.6 \pm 9.9 ) after targeting probe injection and the disseminated tumor nodes $(0.9 \pm 0.5 \mathrm{~mm})$ (blue arrow) can be clearly defined $\mathbf{B}$. The presence of liver tumors as confirmed by bioluminescence imaging $\mathbf{C}$. Surgical guidance by intraoperative FMI-NIR D. The implanted liver tumor tissue $(0.7 \pm 0.3 \mathrm{~mm})(\mathrm{blue}$ arrow) exhibits obvious contrast (TBR: $2.3 \pm 0.5$ ) in color and texture with normal liver tissues E. The merged color and fluorescence image demonstrates the excellent contrast $\mathbf{F}$. Experiments were run in triplicate. 
to be readily eliminated by the liver owing to its size. We determined that the optimal time point for the maximum TBR was $72 \mathrm{~h}$ post-injection for optical imaging. The TBR was $2.6 \pm 0.1$ in the SPIO@Liposome-ICG-RGD group, which was about 2-fold compared to the TBR value of $1.3 \pm 0.1$ in the control group, suggesting that our probes have a high efficiency of tumor targeting ability and were sufficient for intraoperative surgical guidance procedures. In the orthotopic model, the probe was able to assist in obtaining the information of location and size of the tumor preoperatively and to highlight the otherwise readily omitted tiny tumor $(0.6 \pm 0.3 \mathrm{~mm})$ intraoperatively. In the intrahepatic metastasis model, the preoperative assessment was able to aid in staging the liver cancer according to the preoperative MRI scanning image and in the proposal of a reasonable treatment scheme, which would likely have considerable impact on clinical practice.

The ultimate aim of our studies was to improve the practical application of this system. The SPIO@ Liposome-ICG-RGD exhibits an attractive perspective toward clinical application for the following reasons. First, SPIOs represent the first nanoparticle MRI contrast agents used clinically [9], and have excellent biocompatibility and R2 relaxivity. Second, ICG is the only NIR dye approved by the FDA for diagnosis in clinical applications [12]. Furthermore, RGD is a bio-polypeptide, which facilitates its incorporation into the liposomes that are primarily composed of lecithin and cholesterol. Together, these traits suggest a potential wide breadth of clinical application. In addition, both ICG and SPIO are excellent photothermal agents and can be used in the photothermal treatment process $[25,26]$.

\section{MATERIALS AND METHODS}

\section{Cell lines}

The high $\alpha v \beta 3$ receptor-expressing human liver cancer (HepG2) was purchased from the Academy of Military Medical Sciences (China) and cultured in
Dulbecco's modified Eagle's medium (Hyclone, Beijing, China) supplemented with $1 \%$ penicillin/streptomycin (Gibco, China) and $10 \%$ fetal bovine serum (Gibco). The cultured cells were maintained at $37^{\circ} \mathrm{C}$ and $5 \% \mathrm{CO}_{2}$ in a tissue culture incubator.

\section{Animal experiments}

The 6 to 8-week-old Balb/c male nude mice which had HepG2 were obtained from the Laboratory Animal Center of the Chinese Academy of Medical Sciences. All animal experiments were conducted in compliance with the regulations established by the Institutional Animal Care and Use Committee of Southern Medical University. All animal procedures were implemented with isoflurane gas anesthesia ( $3 \%$ isoflurane-air mixture). The subcutaneous liver tumor mice models were established by subcutaneously injecting $5 \times 10^{6} \mathrm{HepG} 2$ cells. The orthotopic liver tumor mice models were established by injecting $5 \times 10^{6}$ cells into the liver via laparotomy. The animals were administered the probes at $10 \mathrm{mg} / \mathrm{kg}$ (total amount of SPIOs) $(\mathrm{n}=5)$ for the in vivo MRI and optical studies.

\section{Synthesis of SPIO@Liposome-ICG-RGD}

Figure 7 illustrates the procedures used for preparing the SPIO@Liposome-ICG-RGD probes. The SPIO cores $\left(\mathrm{Fe}_{3} \mathrm{O}_{4} \mathrm{NPs}\right)$ were synthesized as described previously [27]. The hydrodynamic diameter of the $\mathrm{Fe}_{3} \mathrm{O}_{4}$ NPs was $26 \pm 4 \mathrm{~nm}$ based on dynamic light scattering with a zeta potential of $-20.2 \mathrm{mv}$ (Malvern Instruments Zetasizer Nano ZS, Malvern, UK). The SPIO@Liposome was produced by the film method followed by extrusion as described by Sabate et al. [28] with some modification. Briefly, as an increased content of cholesterol could reduce the uptake of liposomes by the liver [29], we adjusted the soybean lipid phosphatidylcholine and cholesterol, at a molar ratio of $1: 1$, to the amounts required to meet a lipid concentration of $10 \mathrm{mM}$, which was dissolved in a mixture of chloroform and ethanol(volume ratio:3:1).

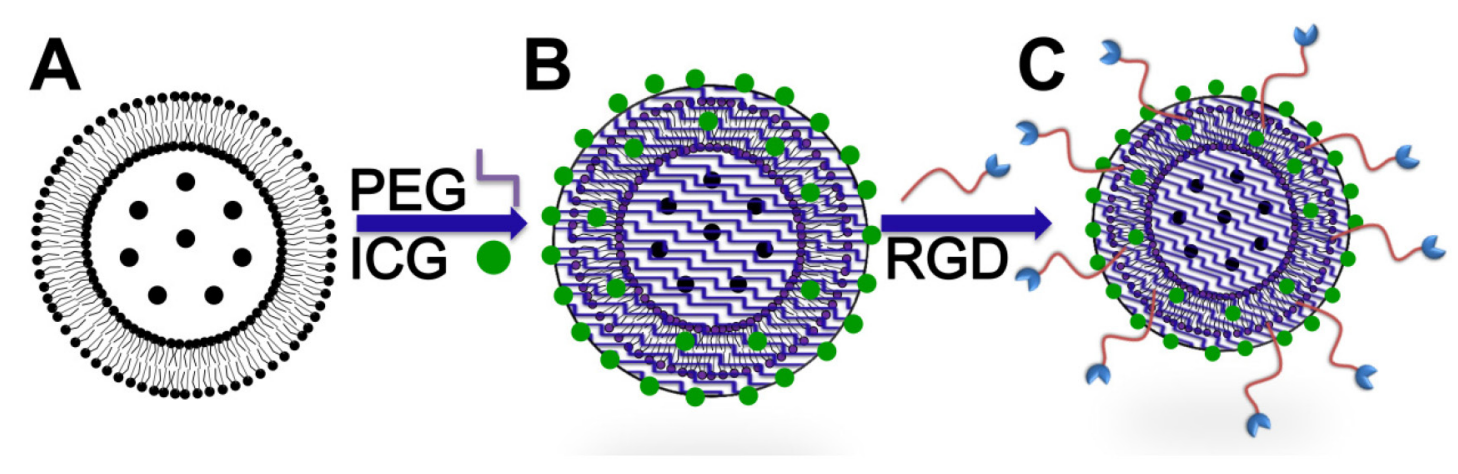

Figure 7: Schematic diagram for the synthesis of SPIO@Liposome-ICG-RGD. A. SPIO nanoparticles was coated with liposome (SPIO@Liposome). B. ICG molecules were loaded into the lipid layer of magnetic liposomes (SPIO@Liposome-ICG). C. RGDs were conjugated to obtain the SPIO@Liposome-ICG-RGD probes. 
The solvent was evaporated with a rotary evaporator (Rotavapor r-144, BUCHI, New Castle, DE, USA) under reduced pressure at $40^{\circ} \mathrm{C}$ and the lipid film was produced in a round bottom flask. After the removal of chloroform, the lipid film was mixed with $250 \mu \mathrm{g} / \mathrm{mL}$ SPIO water suspension to generate a $5 \mathrm{~mL}$ solution. A total of $30 \mathrm{~min}$ of bath sonication was performed prior to extrusion for completing the hydration of the lipid film. The extrusion (ATS Extruder, Cambridge, ON, Canada) was performed 4 times using polycarbonate membranes (Whatman, Maidstone, UK) with a pore diameter of 100 nm. Then, the SPIO@Liposome nanoparticles were coated with polyethylene glycol (PEG) MW-5000 using a dual solvent exchange method. An ICG water solution (25, 50, 75 , and $150 \mathrm{~mL}$ ) at a concentration of $1 \mathrm{mg} / \mathrm{mL}$ was then mixed with $1 \mathrm{mg} / \mathrm{mL}$ SPIO@Liposome for $1 \mathrm{~mL}$ probe sample to make $1 \mathrm{~mL}$. The mixture was then placed on a shaker and incubated overnight at room temperature. The unloaded ICG molecules and SPIOs were removed by ultracentrifugation at 10,000 rpm. The RGD (Arg-GlyAsp, $5 \mathrm{mg}, 98 \%$, USA) peptide was combined with the SPIO@Liposome-ICG nanoparticles using the NHS-EDS method and vibrated for $6 \mathrm{~h}$. The targeted probe was stored at room temperature in silver paper to protect it from light. For in vivo imaging, $250 \mu \mathrm{g}$ SPIO@Liposome-ICG-RGD (total amount of SPIO) was used for each mouse, which contained $12.5 \mu \mathrm{g}$ ICG dye molecules.

\section{Cell cytotoxicity assays}

The HepG2 cells were used for the cytotoxicity assays. The cellular cytotoxicity of the SPIO, SPIO@ Liposome-ICG, and SPIO@Liposome-ICG-RGD probes were evaluated by the 3-(4, 5-dimethylthiazol-2-yl)-2, 5-diphenyltetrazolium bromide (MTT) assay (Promega, Shanghai, China). The HepG2 cells were cultured in 96well microtiter plates at 10,000 cells per well. After 24 $\mathrm{h}$, the cells were washed with phosphate buffered saline (PBS) and the medium was replaced by fresh medium with various concentrations of SPIO, SPIO@Liposome-ICG, orSPIO@Liposome-ICG-RGD (10, 20, 40, 60, 80, and $100 \mu \mathrm{g} / \mathrm{mL}, \mathrm{Fe})$. An equivalent volume of fresh medium (200 $\mu \mathrm{L} /$ well) was added to the control wells. After 24 $\mathrm{h}$ incubation, the treated cells were added to PBS. About $100 \mu \mathrm{L}$ of fresh medium and $10 \mu \mathrm{L}$ MTT solution $(5 \mathrm{mg}$ / $\mathrm{mL}$ in PBS) were added to each well and then cultured for another $4 \mathrm{~h}$. After the removal of the old medium, 120 $\mu \mathrm{L}$ dimethyl sulfoxide was added to each well and the absorbance at $600 \mathrm{~nm}$ was read using a microplate reader to calculate relative cell viabilities.

\section{Integrin binding assays}

To verify the targeted specificity of SPIO@ Liposome-ICG-RGD for HepG2 liver cancer cells, in vitro cell uptake experiments were conducted. Approximately
$1 \times 10^{5}$ HepG2 cells were cultured in $10 \mathrm{~mL}$ of culture medium for $24 \mathrm{~h}$. Then, the SPIO@Liposome-ICG or SPIO@Liposome-ICG-RGD (iron concentration, 0.03 $\mathrm{mM}$ ) was added for another $4 \mathrm{~h}$. After washing three times with cell culture medium and twice with PBS, another 5 $\mathrm{mL}$ cell culture medium was added. The NIR fluorescence images (ProEM 1024B Excelon, Princeton Instruments, Trenton, NJ, USA) were acquired by the electronmultiplying CCD (EMCCD) and the noise to background ratio was analyzed [30]. In addition, the iron content per cell was analyzed using inductively coupled plasma mass spectrometry (ICP-MS) in the same sample. The number of cells was obtained using a Neubauer counting chamber.

\section{In vivo tumor fluorescence imaging}

In vivo fluorescence imaging was performed on ten mice with subcutaneous liver tumors $>5 \mathrm{~mm}$ in diameter. About $250 \mu \mathrm{L}$ SPIO@Liposome-ICG or SPIO@Liposome-ICG-RGD ( $\mathrm{n}=5$ /group) was delivered by the tail vein. The IVIS Spectrum Imaging System (PerkinElmer, Rodgau, Germany) was used to monitor the change in the fluorescence signal of the mice at 10 min and at 12, 24, 48, 72, 96, and $120 \mathrm{~h}$ post-injection with excitation and emission wavelengths of $780 \mathrm{~nm}$ and $830 \mathrm{~nm}$, respectively. Imaging data were analyzed with IVIS Living Image 3.0 software. The region of interest (ROI) was selected for measuring the mean fluorescence intensity (MFI) of the tumors. The TBR was acquired by the MFI of the tumor area divided by the corresponding body background area.

\section{In vivo tumor MR imaging}

The in vivo MRI tests were performed on a 1.0-T MRI system (Aspect, Shoham, Israel). The T2-weighted fast spin echo imaging sequence with a TR of $2394 \mathrm{~ms}$, TE of $22.2 \mathrm{~ms}$, field of view of $30 \mathrm{~mm} \times 30 \mathrm{~mm}$, and slice thickness of $1 \mathrm{~mm}$ was used to obtain MR images. The MRI contrast change in the tumor following injection of the targeted probe was quantitatively calculated using the ROI method with Mxliteview (Philips). Averaged MRI signal intensities of the ROI were calculated from the tumor and corresponding noise areas. The normalized signal intensities (SI) were obtained by comparing SIpre and SIpost of the tumor ROI. The mean MRI contrast change was calculated from five MRI image slices of the entire tumor.

\section{Probe biodistribution and histologic examination}

The mice were sacrificed following optical/ MRI imaging. The main organs (heart, liver, spleen, lung, and kidney) and the tumors were collected for the biodistribution of probes and histological analysis. The optical images for assessing the fluorescence distribution were obtained using the IVIS Spectrum system. Prussian 
blue staining was performed for the detection of iron in the tissue sections. Tissue morphology was verified by hematoxylin and eosin (HE) staining. The slices were examined using a digital microscope (Leica QWin, Wetzlar, Germany).

\section{Statistical analyses}

All data were presented as the means \pm standard deviation from at least a three sample replicates. Statistical comparisons were conducted using a Student's t-test or paired t-test and GraphPad Prism 5 software (LaJolla, CA, USA). A statistically significant difference was defined as a value of $\mathrm{P}<0.05$.

\section{CONFLICTS OF INTEREST}

The authors declare no competing financial interests.

\section{FINANCIAL SUPPORT}

This work was supported by the National Basic Research Program of China (973 Program) under Grant No. 2015CB755500, the National Natural Science Foundation of China under Grant Nos. 81227901, 61231004, 81501540, 61401462, 61501462, 81527805, and 81470083, the National High Technology Research and Development Program of China (863 Program) under Grant No. 2012AA021105, the National Natural Science Foundation of China-Guangdong United Funds (Grant No.U1401254), and the "Climbing Program" Special Funds:No. pdjh2016a0090, pdjh2016b0103, Guangdong province science and technology plan project No.2016A020220013. Digital Theranostic Equipment Research Special program of The "13th five-year" National Key Research Plan (No.2016YFC0106500), The Major Instrument Project of National Natural Science Fund (No.81627805), The Science and Technology Plan Project of Guangzhou (No.201604020144) .

\section{REFERENCES}

1. American Cancer Society. Global Cancer Facts \& Figures 3rd Edition 2012, http://www.cancer.org/research/ cancerfactsstatistics/global.

2. American Cancer Society. Cancer Facts \& Figures 2013, http://www.cancer.org/research/cancerfactsstatistics/global.

3. Yang JD, Roberts LR. Hepatocellular carcinoma: A global view. Nat Rev Gastroenterol Hepatol. 2010; 7:448-58. doi: 10.1038/nrgastro.2010.100.

4. Fan ST. Hepatocellular carcinoma--resection or transplant? Nat Rev Gastroenterol Hepatol. 2012; 9:732-37. doi: 10.1038/nrgastro.2012.158.

5. Vilgrain V, Van Beers BE, Pastor CM. Insights into the diagnosis of hepatocellular carcinomas with hepatobiliary
MRI. J Hepatol. 2016; 64:708-16. doi: 10.1016/j. jhep.2015.11.016.

6. Nguyen QT, Tsien RY. Fluorescence-guided surgery with live molecular navigation--a new cutting edge. Nat Rev Cancer. 2013; 13:653-62. doi: 10.1038/nrc3566.

7. Brooks PC, Clark RA, Cheresh DA. Requirement of vascular integrin alpha $\mathrm{v}$ beta 3 for angiogenesis. Science. 1994; 264:569-71. doi: 10.1126/science.7512751.

8. Semela D, Dufour JF. Angiogenesis and hepatocellular carcinoma. J Hepatol. 2004; 41:864-80. doi: 10.1016/j. jhep.2004.09.006.

9. Na HB, Song IC, Hyeon T. Inorganic nanoparticles for MRI contrast agents. Adv Mater. 2009; 21:2133-48. doi: 10.1002/adma.200802366.

10. Lee N, Hyeon T. Designed synthesis of uniformly sized iron oxide nanoparticles for efficient magnetic resonance imaging contrast agents. Chem Soc Rev. 2012; 41:2575-89. doi: 10.1039/C1CS15248C.

11. Brähler M, Georgieva R, Buske N, Müller A, Müller S, Pinkernelle J, Teichgräber U, Voigt A, Bäumler H. Magnetite-loaded carrier erythrocytes as contrast agents for magnetic resonance imaging. Nano Lett. 2006; 6:2505-09. doi: 10.1021/n10618501.

12. Maarek JM, Holschneider DP, Harimoto J. Fluorescence of indocyanine green in blood: intensity dependence on concentration and stabilization with sodium polyaspartate. J Photochem Photobiol B. 2001; 65:157-64. doi: 10.1016/ S1011-1344(01)00264-0.

13. Sun T, Zhang YS, Pang B, Hyun DC, Yang M, Xia Y. Engineered nanoparticles for drug delivery in cancer therapy. Angew Chem Int Ed Engl. 2014; 53:12320-64.

14. Bu L, Xie J, Chen K, Huang J, Aguilar ZP, Wang A, Sun KW, Chua MS, So S, Cheng Z, Eden HS, Shen B, Chen X. Assessment and comparison of magnetic nanoparticles as MRI contrast agents in a rodent model of human hepatocellular carcinoma. Contrast Media Mol Imaging. 2012; 7:363-72. doi: 10.1002/cmmi.494.

15. Chi C, Du Y, Ye J, Kou D, Qiu J, Wang J, Tian J, Chen X. Intraoperative imaging-guided cancer surgery: from current fluorescence molecular imaging methods to future multimodality imaging technology. Theranostics. 2014; 4:107284. doi: 10.7150/thno.9899.

16. Boonstra MC, van Driel PB, van Willigen DM, Stammes MA, Prevoo HA, Tummers QR, Mazar AP, Beekman FJ, Kuppen PJ, van de Velde CJ, Löwik CW, Frangioni JV, van Leeuwen FW, et al. uPAR-targeted multimodal tracer for pre- and intraoperative imaging in cancer surgery. Oncotarget. 2015; 6:14260-73. doi: 10.18632/ oncotarget.3680.

17. Ding J, Wang Y, Ma M, Zhang Y, Lu S, Jiang Y, Qi C, Luo S, Dong G, Wen S, An Y, Gu N. CT/fluorescence dual-modal nanoemulsion platform for investigating atherosclerotic plaques. Biomaterials. 2013; 34:209-16. doi: 10.1016/j.biomaterials.2012.09.025. 
18. Sampath L, Kwon S, Hall MA, Price RE, Sevick-Muraca EM. Detection of Cancer Metastases with a Dual-labeled Near-Infrared/Positron Emission Tomography Imaging Agent. Transl Oncol. 2010; 3:307-217. doi: 10.1593/ tlo.10139.

19. Vahrmeijer AL, Hutteman M, van der Vorst JR, van de Velde CJ, Frangioni JV. Image-guided cancer surgery using near-infrared fluorescence. Nat Rev Clin Oncol. 2013; 10:507-18. doi: 10.1038/nrclinonc.2013.123.

20. Gao W, Ji L, Li L, Cui G, Xu K, Li P, Tang B. Bifunctional combined $\mathrm{Au}-\mathrm{Fe}(2) \mathrm{O}(3)$ nanoparticles for induction of cancer cell-specific apoptosis and real-time imaging. Biomaterials. 2012; 33:3710-18. doi: 10.1016/j. biomaterials.2012.01.047.

21. Qi H, Li Z, Du K, Mu K, Zhou Q, Liang S, Zhu W, Yang $\mathrm{X}$, Zhu Y. Transferrin-targeted magnetic/fluorescence micelles as a specific bi-functional nanoprobe for imaging liver tumor. Nanoscale Res Lett. 2014; 9:595. doi: 10.1186/1556-276X-9-595.

22. Desgrosellier JS, Cheresh DA. Integrins in cancer: biological implications and therapeutic opportunities. Nat Rev Cancer. 2010; 10:9-22. doi: 10.1038/nrc2748.

23. Feron O. Tumor-penetrating peptides: a shift from magic bullets to magic guns. Sci Transl Med. 2010; 2:34ps26. doi: 10.1126/scitranslmed.3001174.

24. Carvalho A, Martins MB, Corvo ML, Feio G. Enhanced contrast efficiency in MRI by PEGylated magnetoliposomes loaded with PEGylated SPION: effect of SPION coating and micro-environment. Mater Sci Eng C. 2014; 43:521-26. doi: 10.1016/j.msec.2014.07.055.

25. Ma Y, Tong S, Bao G, Gao C, Dai Z. Indocyanine green loaded SPIO nanoparticles with phospholipidPEG coating for dual-modal imaging and photothermal therapy. Biomaterials. 2013; 34:7706-14. doi: 10.1016/j. biomaterials.2013.07.007.

26. Mundra V, Peng Y, Rana S, Natarajan A, Mahato RI. Micellar formulation of indocyanine green for phototherapy of melanoma. J Control Release. 2015; 220:130-40. doi: 10.1016/j.jconrel.2015.10.029.

27. Wu Y, Song M, Xin Z, Zhang X, Zhang Y, Wang C, Li S, $\mathrm{Gu}$ N. Ultra-small particles of iron oxide as peroxidase for immunohistochemical detection. Nanotechnology. 2011; 22:225703. doi: 10.1088/0957-4484/22/22/225703.

28. Sabaté R, Barnadas-Rodríguez R, Callejas-Fernández J, Hidalgo-Alvarez R, Estelrich J. Preparation and characterization of extruded magnetoliposomes. Int J Pharm. 2008; 347:156-62. doi: 10.1016/j.ijpharm.2007.06.047.

29. Patel HM, Tuzel NS, Ryman BE. Inhibitory effect of cholesterol on the uptake of liposomes by liver and spleen. Biochim Biophys Acta. 1983; 761:142-51. doi: 10.1016/0304-4165(83)90223-4.

30. Chi C, Ye J, Ding H, He D, Huang W, Zhang GJ, Tian J. Use of indocyanine green for detecting the sentinel lymph node in breast cancer patients: from preclinical evaluation to clinical validation. PLoS One. 2013; 8:e83927. doi: 10.1371/journal.pone.0083927. 